\title{
Third-generation antibody drug conjugates for cancer therapy - a balancing act
}

\author{
"Globally, with new payloads and evolving linkers, taking a \\ holistic approach to the development of antibody-drug conjugates \\ remains paramount.
}

First draft submitted: 11 Jan 2016; Accepted for publication: 13 Jan 2016; Published online: 19 February 2016

Keywords: ADC • antibody-targeted drug delivery • cancer therapy • payload $\bullet$ site-specific conjugation

\section{Historical perspective}

The use of monoclonal antibodies (mAbs) to target toxic payloads to cancer cells has a long and convoluted history. Early experiments with first-generation antibody-drug conjugates (ADCs) consisting of conventional chemotherapy linked to target-specific mAbs underwhelmed due to insufficient potency of the payload and/or toxicity due to instability of the ADC and systemic loss of the drug. Second-generation ADCs used more potent (100-1000-fold) tubulin-targeting agents such as the auristatins (monomethyl auristatin E [MMAE], monomethyl auristatin $\mathrm{F}$ [MMAF]) and maytansinoids (DM1, DM4) or DNA-targeting agents such as the calicheamicins, and led to the relatively recent US FDA approval of two microtubule-inhibiting ADCs: brentuximab vedotin (SGN-35, Acetris ${ }^{\circledR}$, 2011) for Hodgkin and anaplastic large cell lymphoma and ado-trastuzumab emtansine (T-DM1, Kadcyla ${ }^{\circledR}$, 2013) for metastatic HER2-positive breast cancer. These approvals have led to an explosion in ADC R\&D, with currently over 50 ADCs undergoing clinical evaluation [1].

\section{Remaining challenges of second- generation ADCs}

The second-generation ADCs are not without remaining challenges. Notably, the heterogeneity of the final ADC with respect to drug-antibody ratio (DAR) due to stochastic coupling strategies, the narrow therapeutic index, the limited penetration of solid tumors and the development of resistance continue to drive the field toward further improvements. 'Traditional' chemical conjugation of payload to the mAbs occurs through solventexposed lysine residues (via succinimide ester derivatization) or via interchain cysteine residues (maleimide chemistry) resulting in both cases in a distribution of DARs (0-8) with an average of 3-4, combined with coupling site heterogeneity. ADCs species with DAR $>4$ have an increased tendency to aggregate, negatively impacting in vivo characterization and clearance rates.

The relationship between drug-linker characteristics, serum stability and therapeutic index of ADCs in humans is complex and not always mirrored by preclinical rodent models [1-3]. ADC linkers are broadly classified into cleavable: through disulfide reduction by intracellular glutathione (N-succinimidyl-4-[2-pyridyldithio] pentanoate [SPP]; N-succinimidyl-4-[2-pyridyldithio] butanoate [SPDB]), via acid-labile hydrazone linkers [AcBut]), or through peptidase activity (valine-citrulline dipeptide [VC]), and noncleavable: mainly thioether linkers (N-succinimidyl-4-[N-maleimidomethyl] cyclohexane-1-carboxylate [MCC]) where the hydrophilic nature of the intracellular metabolites precludes diffusion into the extracellular environment and ensuing 'bystander' killing. ADCs with noncleavable linkers exhibit increased serum stability in
Mireille Vankemmelbeke ${ }^{*}, 1$ \& Lindy Durrant ${ }^{1}$

'Division of Cancer \& Stem Cells, School of Medicine, City Hospital Campus, University of Nottingham, Hucknall Road, Nottingham, NG5 1PB, UK *Author for correspondence: mireille.vankemmelbeke@ nottingham.ac.uk 
rodents with reduced hepatic metabolite detoxification, which is evident from a reduced weight loss compared with cleavable linkers. In humans, the picture is less clear with divergent dose-limiting toxicities (DLT) depending on linker stability and more labile linkers being better tolerated. Additionally, the hydrophobicity and spacer length of the linker also affect linker stability, clearance rates and interaction with multidrug resistance transporters.

Interestingly, in vivo toxicity with second-generation ADCs is frequently not target-specific. Clinical toxicity comprises hepatotoxicity, peripheral neuropathy, thrombocytopenia and ocular toxicity, with ADCs against divergent targets, but with common druglinker design, sharing toxicities suggesting a contribution by the nature of the drug-linker. In particular, cell-permeable tubulin inhibitors with cleavable linkers (SPDB-DM4 or VC-MMAE) frequently cause peripheral neuropathy, whereas noncleavable linkers often trigger hematological toxicity, possibly due to increased dosing and interactions with $\mathrm{F} \gamma \gamma$ receptors on hematopoietic cells. Adverse ocular events seem to be common to both types of linkers and payloads, suggesting that sustained overall tubulin inhibitor exposure is the determining factor.

\section{Third-generation ADCs raise hope for an increased therapeutic window}

The aforementioned concerns associated with secondgeneration ADCs have expedited the introduction of third-generation ADCs. Considerable emphasis is now being placed on site-specific conjugation in order to ensure homogenous ADCs with well-defined DARs, coinciding with an expansion of the payload arsenal including drugs with novel modes of action and a wider dynamic cytotoxicity range. The breadth of sitespecific conjugation technologies encompasses chemoenzymatic conjugation through the introduction of amino acids or via glycosylation adducts. The need for $\mathrm{mAb}$ re-engineering in some of these approaches, however, can impact on development time.

The site-specific introduction of single cysteine residues at strategic positions (THIOMAB) was first out of the starting blocks generating ADCs with DAR of two with an improved safety profile and maintenance of efficacy, compared with traditionally conjugated ADCs with higher DARs [4]. Crucially, conjugation site solvent accessibility and linker stability were inversely correlated with sites with intermediate accessibility displaying better in vivo efficacy and safety due to increased stability, highlighting the importance of optimal site selection [5]. An alternative strategy without the need for $\mathrm{mAb}$ engineering comprises the ThioBridge approach [6]. Interchain disulfides (four per $\mathrm{mAb}$ ) are reduced and rebridged with the drug incorporated into the bridge generating a near homogenous ADC with DAR 4 and increased serum stability. Bio-orthogonal chemistry using amber stop codons in order to introduce unnatural amino acids such as p-acetylphenylalanine or the naturally occurring selenocysteine for drug conjugation are alternative sitespecific strategies [7].

Finally, enzymatic drug conjugation combined with strategically placed sequence tags or glycans are being explored. The formyl glycine generating enzyme converts the cysteine in the CXPXR sequence tag to a formyl glycine with a reactive aldehyde group (SMARTagTM) [8]. An alternative approach employs microbial transglutaminase and a XLLQGX sequence tag to crosslink glutamine side chains to free amines (TG-ADCTM). An improvement of this method consists of exploiting the naturally occurring glutamine residue at position 295 and/or 297 (via N to Q substitution) after deglycosylation. The need for deglycosylation was offset by a homogenous DAR of 4, equivalent efficacy compared with a conventionally conjugated ADC and improved pharmacokinetics (PK) and therapeutic index in vivo [9]. Bacterial sortase activity combined with a carboxyterminal LPETG recognition motif and a pentaglycine-linked drug led to a homogenously labelled ADC with DAR 2 (SMAC$\mathrm{TAG}^{\mathrm{TM}}$ ) [10]. Glycosyltransferases join the battery of enzymes being exploited in order to incorporate reactive groups (e.g., 6-thiofucose) at position 297 [11].

Preclinical comparison of site-specific ADCs with homogenous DARs with stochastically conjugated ADCs revealed similar efficacy and PK parameters in cases where a site-specific DAR of 2 was evaluated. In contrast, ADC conjugations where a site-specific DAR of 4 was achieved, led to superior PK and efficacy compared with a stochastically conjugated ADC [9,12]. Recently, even higher homogenous drug loading (DAR 8) with superior efficacy was achieved through careful management of drug-linker hydrophobicity [13]. Crucially, whether this will deliver on the wider therapeutic index promise in the clinic remains to be evaluated.

At the same time, efforts are continuing to expand on payloads with novel modes of action with a focus on agents with activity against nonproliferating cancer cells in order to widen the target area to include tumor-initiating cells (TICs) and to overcome resistance. Furthest in development are pyrrolobenzodiazepines (PBDs), derivatives of naturally occurring tricyclic antibiotics that form interstrand crosslinks or alkylate single DNA strands causing cell death with (sub) picomolar $\mathrm{EC}_{50}$ [14]. Currently, four PBDcontaining ADCs are undergoing clinical evaluation, with the alkylating monoimine containing indolino- 
PBDs being better tolerated than the diimine containing crosslinking PBDs, the latter of which exhibit late-onset myelosuppression and hematological DLT. Preclinically, the PBD-containing ADC, SC16LD6.5, was recently shown to eliminate pulmonary neuroendocrine TICs in vivo [15]. ADCs containing topoisomerase inhibitors such as $\mathrm{SN}-38$ (an irinotecan metabolite with enhanced activity) have also progressed significantly and are currently undergoing clinical evaluation. Intriguingly, IMMU-132, with an average DAR of 7.6 and a relatively labile linker, displayed no apparent detrimental PK effects, although neutropenia was the DLT [16]. Other payloads with cell cycle-independent activity comprise the duocarmycins [17,18] and anthracyclines (nemorubicin and its more potent metabolite PNU-159682), the latter of which recently being used to target TICs in colon cancer (NMS818 [19]) and, critically, have been shown to overcome resistance to microtubule-targeting compounds (NMS249 [20]). Finally, $\alpha$-amanitin, a bicyclic octapeptide from the fungus Amanita, is noteworthy as this RNA polymerase II inhibitor constitutes a nonDNA targeting drug, which in an ADC format led to potent preclinical antitumor efficacy [21]. This expanding array of payloads introduces the possibility of combination warhead ADCs.

\section{Considerations \& outlook}

Preclinical evaluation of the recent wave of third-generation site-specifically and homogenously conjugated ADCs has offered reasons for optimism in the ADC field, but at the same time, as relatively few of those ADCs have reached the clinic, caution remains over

\section{References}

1 Polakis P. Antibody-drug conjugates for cancer therapy. Pharmacol. Rev. 68(1), 3-19 (2016).

2 Drake PM, Rabuka D. An emerging playbook for antibodydrug conjugates: lessons from the laboratory and clinic suggest a strategy for improving efficacy and safety. Curr. Opin. Chem. Biol. 28, 174-180 (2015).

3 Hong EE, Erickson H, Lutz RJ et al. Design of coltuximab ravtansine, a CD19-targeting antibody-drug conjugate (ADC) for the treatment of B-Cell malignancies: structureactivity relationships and preclinical evaluation. Mol. Pharmaceutics 12(6), 1703-1716 (2015).

4 Panowksi S, Bhakta S, Raab H, Polakis P, Junutula JR. Sitespecific antibody drug conjugates for cancer therapy. $m A b s$ 6(1), 34-45 (2014).

5 Shen BQ, Xu K, Liu L et al. Conjugation site modulates the in vivo stability and therapeutic activity of antibody-drug conjugates. Nature Biotechnol. 30 (2), 184-189 (2012).

6 Badescu G, Bryant P, Bird M et al. Bridging disulfides for the continuing narrow therapeutic margin. On-target efficacy driven by sustained exposure is aided by the relatively long half-life of ADCs with full-length $\mathrm{mAbs}$, but their limited penetration of solid tumors is opening the door to smaller $\mathrm{mAb}$ fragments, perhaps with higher DAR. This will inevitably impact on half-life, but their faster clearance may aid tolerability if drug and linker are carefully tailored [13]. Targeting TICs with cell cycleindependent payloads is also showing promise, but critical appraisal of cancer stem cell 'markers' is vital in order to avoid on-target toxicity due to normal tissue crossreactivity [19]. Globally, with new payloads and evolving linkers, taking a holistic approach to the development of ADCs remains paramount.

Finally, as cancer immunotherapy is penetrating all aspects of cancer drug development, a recent finding suggests that there is also potential for immunemediated effects in the ADC field, with T-DM1 treatment of Her2+ early breast cancer eliciting antitumor immune responses that could be potentiated with antiCTLA-4/anti-PD1 combination therapy, opening the door to combination strategies of checkpoint blockade with ADCs [22].

\section{Financial \& competing interests disclosure}

The authors have no relevant affiliations or financial involvement with any organization or entity with a financial interest in or financial conflict with the subject matter or materials discussed in the manuscript. This includes employment, consultancies, honoraria, stock ownership or options, expert testimony, grants or patents received or pending, or royalties.

No writing assistance was utilized in the production of this manuscript.

stable and defined antibody drug conjugates. Bioconjugate chemistry 25(6), 1124-1136 (2014).

7 Hallam TJ, Wold E, Wahl A, Smider VV. Antibody conjugates with unnatural amino acids. Mol. Pharmaceutics 12(6), 1848-1862 (2015).

8 Drake PM, Albers AE, Baker J et al. Aldehyde tag coupled with HIPS chemistry enables the production of ADCs conjugated site-specifically to different antibody regions with distinct in vivo efficacy and PK outcomes. Bioconjugate Chem. 25(7), 1331-1341 (2014).

9 Lhospice F, Bregeon D, Belmant C et al. Site-specific conjugation of monomethyl auristatin $\mathrm{E}$ to anti-CD30 antibodies improves their pharmacokinetics and therapeutic index in rodent models. Mol.Pharmaceutics 12(6), 18631871 (2015).

10 Beerli RR, Hell T, Merkel AS, Grawunder U. Sortase enzyme-mediated generation of site-specifically conjugated antibody drug conjugates with high in vitro and in vivo potency. PloS One 10(7), e0131177 (2015).

11 Okeley NM, Toki BE, Zhang X et al. Metabolic engineering of monoclonal antibody carbohydrates for antibody-drug 
conjugation. Bioconjugate Chem. 24(10), 1650-1655 (2013).

12 Bryant $\mathrm{P}$, Pabst $\mathrm{M}$, Badescu $\mathrm{G}$ et al. In vitro and in vivo evaluation of cysteine rebridged trastuzumab-MMAE antibody drug conjugates with defined drug-to-antibody ratios. Molecular Pharmaceutics 12(6), 1872-1879 (2015).

13 Lyon RP, Bovee TD, Doronina SO et al. Reducing hydrophobicity of homogeneous antibody-drug conjugates improves pharmacokinetics and therapeutic index. Nature Biotechnol. 33(7), 733-735 (2015).

14 Rahman KM, Jackson PJ, James CH et al. GC-targeted C8-linked pyrrolobenzodiazepine-biaryl conjugates with femtomolar in vitro cytotoxicity and in vivo antitumor activity in mouse models. J. Med. Chem. 56(7), 2911-2935 (2013).

15 Saunders LR, Bankovich AJ, Anderson WC et al. A DLL3targeted antibody-drug conjugate eradicates high-grade pulmonary neuroendocrine tumor-initiating cells in vivo. Sci. Transl. Med. 7(302), 302ra136 (2015).

16 Goldenberg DM, Cardillo TM, Govindan SV, Rossi EA, Sharkey RM. Trop-2 is a novel target for solid cancer therapy with sacituzumab govitecan (IMMU-132), an antibodydrug conjugate (ADC). Oncotarget 6(26), 22496-22512 (2015).

17 Van Der Lee MM, Groothuis PG, Ubink R et al. The preclinical profile of the duocarmycin-based HER2-targeting
ADC SYD985 predicts for clinical benefit in low HER2expressing breast cancers. Mol. Cancer Therap. 14(3), 692-703 (2015).

18 Owonikoko TK, Hussain A, Stadler WM et al. First-inhuman multicenter Phase I study of BMS-936561 (MDX1203), an antibody-drug conjugate targeting CD70. Cancer Chemotherapy Pharmacol. doi:10.1007/s00280-015-2909-2 (2015) (Epub ahead of print).

19 Junttila MR, Mao W, Wang X et al. Targeting LGR5 + cells with an antibody-drug conjugate for the treatment of colon cancer. Sci. Transl. Med. 7(314), 314ra186 (2015).

20 Yu SF, Zheng B, Go M et al. A novel anti-CD22 anthracycline-based antibody-drug conjugate (ADC) that overcomes resistance to auristatin-based ADCs. Clin. Cancer Res. 21(14), 3298-3306 (2015).

21 Moldenhauer G, Salnikov AV, Luttgau S, Herr I, Anderl J, Faulstich $\mathrm{H}$. Therapeutic potential of amanitin-conjugated anti-epithelial cell adhesion molecule monoclonal antibody against pancreatic carcinoma. J. Natl Cancer Institute 104(8), 622-634 (2012).

22 Muller P, Kreuzaler M, Khan T et al. Trastuzumab emtansine (T-DM1) renders HER2 + breast cancer highly susceptible to CTLA-4/PD-1 blockade. Sci. Transl. Med. 7(315), 315ra188 (2015). 